\title{
An interactive simulation tool for patient-specific clinical decision support in single-ventricle physiology
}

Timothy Conover, $\mathrm{PhD},{ }^{\mathrm{a}}$ Anthony M. Hlavacek, $\mathrm{MD},{ }^{\mathrm{b}}$ Francesco Migliavacca, $\mathrm{PhD},{ }^{\mathrm{c}}$ Ethan Kung, $\mathrm{PhD},{ }^{\mathrm{a}}$ Adam Dorfman, MD, ${ }^{\mathrm{d}}$ Richard S. Figliola, PhD, ${ }^{\mathrm{a}}$ and Tain-Yen Hsia, MD, MSc, ${ }^{\mathrm{e}}$ for the Modeling of Congenital Hearts Alliance (MOCHA) Investigators

\section{ABSTRACT}

Objective: Modeling of single-ventricle circulations has yielded important insights into their unique flow dynamics and physiology. Here we translated a state-of-the-art mathematical model into a patient-specific clinical decision support interactive Web-based simulation tool and show validation for all 3 stages of single-ventricular palliation.

Methods: Via the adoption a validated lumped parameter method, complete cardiovascular-pulmonary circulatory models of all 3 stages of single-ventricle physiology were created within a simulation tool. The closed-loop univentricular heart model includes scaling for growth and respiratory effects, and typical patient-specific parameters are entered through an intuitive user interface. The effects of medical or surgical interventions can be simulated and compared. To validate the simulator, patient parameters were collected from catheterization reports. Four simulator outputs were compared against catheterization findings: pulmonary to systemic flow ratio (Qp:Qs), systemic arterial saturation $(\mathrm{SaO} 2)$, mean pulmonary arterial pressure (mPAp), and systemic-venous oxygen difference ( $\mathrm{SaO} 2-\mathrm{SvO} 2)$.

Results: Data from 60 reports were used. Compared with the clinical values, the simulator results were not significantly different in mean Qp:Qs, SaO2, or mPAp $(P>.09)$. There was a statistical but clinically insignificant difference in average $\mathrm{SaO}-\mathrm{SvO} 2$ (average difference $1 \%, P<.01$ ). Linear regression analyses revealed a good prediction for each variable $\left(\mathrm{Qp}: \mathrm{Qs}, \mathrm{R}^{2}=0.79 ; \mathrm{SaO} 2, \mathrm{R}^{2}=0.64 ; \mathrm{mPAp}\right.$, $\left.\mathrm{R}^{2}=0.69 ; \mathrm{SaO} 2-\mathrm{SvO} 2, \mathrm{R}^{2}=0.93\right)$.

Conclusions: This simulator responds quickly and predicts patient-specific hemodynamics with good clinical accuracy. By predicting postoperative and postintervention hemodynamics in all 3 stages of single-ventricle physiology, the simulator could assist in clinical decision-making, training, and consultation. Continuing model refinement and validation will further its application to the bedside. (J Thorac Cardiovasc Surg 2018;155:712-21)

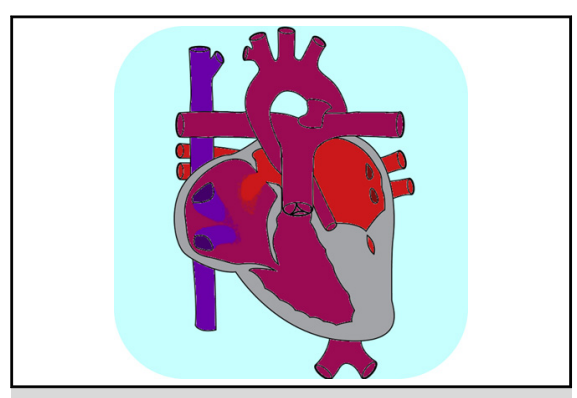

Icon for the iOS app.

\section{Central Message}

A predictive modeling simulation tool for single-ventricle palliations provides comparative hemodynamic and physiologic information for clinical training and to assist in clinical management.

\section{Perspective}

Managing single-ventricle physiology remains challenging. Mathematical models have elucidated the effects of surgery and intervention in all 3 stages of single-ventricle circulations. We describe a Web-based simulator that gives hemodynamic and physiologic information to assist decision-making for clinicians anywhere. This decision support tool is validated against patient-specific catheterization data.

See Editorial Commentaries pages 722 and 724.
Single-ventricle physiology (SVP) represents a multistage surgical palliative pathway with 2 transitional circulations and 1 permanent circulation, each with distinctly modified

\footnotetext{
From ${ }^{\text {a}}$ Department of Mechanical Engineering, Clemson University, Clemson, SC; ${ }^{b}$ Division of Pediatric Cardiology, Medical University of South Carolina,

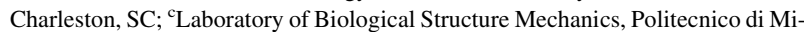
lano, Milan, Italy; ${ }^{\mathrm{d}}$ Division of Pediatric Cardiology, C.S. Mott Children's Hospital, Ann Arbor, Mich; and ${ }^{\mathrm{e}}$ Cardiac Unit, Great Ormond Street Hospital for Children NHS Foundation Trust, London, United Kingdom.

This work received funding support from Leducq Foundation (France).

MOCHA Investigators: Andrew Taylor, Sachin Khambadkone, Silvia Schievano, Marc de Leval, and T.-Y. Hsia (Institute of Cardiovascular Sciences, London, United Kingdom); Edward Bove and Adam Dorfman (University of Michigan, Ann Arbor, Mich); G. Hamilton Baker and Anthony Hlavacek (Medical University of South Carolina, Charleston, SC); Francesco Migliavacca, Giancarlo Pennati, and Gabriele Dubini (Politecnico di Milano, Milan, Italy); Alison Marsden (Stan-
}

hemodynamics and physiologies. In addition to a variety of congenital cardiac defects that require the SVP strategy, different operative options exist at each stage depending on

ford University, Calif); Irene Vignon-Clementel (National Institute of Research in Informatics and Automation, Paris, France); and Richard Figliola and John McGregor (Clemson University, Clemson, SC).

Drs Conover and Hlavacek are co-first authors.

Drs Figliola and Tain-Yen Hsia are co-senior authors.

Received for publication Oct 13, 2016; revisions received Aug 20, 2017; accepted for publication Sept 10, 2017; available ahead of print Oct 20, 2017.

Address for reprints: Tain-Yen Hsia, MD, MSc, Cardiac Unit 7th Floor, Nurses Home, Great Ormond Street Hospital for Children NHS Trust, London WC1N 3JH,

United Kingdom (E-mail: hsiat@gosh.nhs.uk). 0022-5223/\$36.00

Copyright (c 2017 by The American Association for Thoracic Surgery https://doi.org/10.1016/j.jtcvs.2017.09.046 

Abbreviations and Acronyms
BSA = body surface area
$\mathrm{CFD}=$ computational fluid dynamics
$\mathrm{HR}=$ heart rate
LPM = lumped parameter network model
MAP $=$ mean arterial pressure
mBTS $=$ modified Blalock-Taussig shunt
MET $=$ metabolic equivalent
mPAp $=$ mean pulmonary artery pressure
PVR = pulmonary vascular resistance
PVRI = indexed pulmonary vascular resistance
$\mathrm{Qp}: \mathrm{Qs}=$ pulmonary to systemic flow ratio
Qs $\quad=$ systemic blood flow
$\mathrm{RV}-\mathrm{PA}=$ right ventricle to pulmonary artery
$\mathrm{SaO} 2=$ systemic arterial saturation
SAP $=$ single atrial pressure
$\mathrm{SvO} 2=$ systemic venous saturation
SVP = single-ventricle physiology
SVR = systemic vascular resistance
SVRI = indexed systemic vascular resistance
TCPC $=$ total cavopulmonary connection
$\mathrm{vO} 2=$ resting oxygen consumption

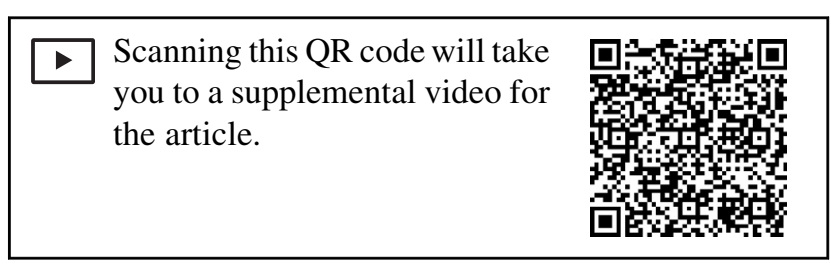

anatomic/physiologic substrate and institutional/surgeon preferences. Nonetheless, despite considerable advances in management of patients with SVP, patients continue to face significant morbidity and mortality throughout their lifetimes. ${ }^{1}$ Each child's unique anatomy and physiology require an individualized approach. Important parameters that regulate pressure and flow, such as pulmonary vascular resistance (PVR), can change dramatically as the patient progresses from neonate to adult, compounding interventional decisions. In this paper, we report the development of a detailed simulation tool based on a simplified modeling technique that can be individualized to each patient to study potential postoperative physiological outcomes for the 3 stages of single-ventricle palliation either at the bedside or in an education environment.

Various research groups have used computational and mathematical modeling to better understand the unique and unnatural features of SVP and to examine the influence of surgical techniques and medical interventions for managing these patients..$^{2-7}$ One approach adopted over the last decade has combined the strengths of computational fluid dynamics (CFD) with lumped parameter network models (LPMs) to allow the comprehensive assessment of hemodynamic effects of the local surgical domain (such as power loss in the total cavopulmonary connection [TCPC]) and impact on the global physiology (such as systemic oxygen delivery). In such multiscale models, CFD predicts the detailed local hemodynamics, including local velocity vector fields, whereas the LPM predicts the systems-level responses, such as flow rates and pressures under appropriate patient conditions.

Previously, we have used these multiscale models to evaluate multiple clinically significant issues and concepts in patients with SVP, such as the hybrid procedure for hypoplastic left heart syndrome, ${ }^{8}$ branch pulmonary artery stenosis, ${ }^{9}$ residual coarctation, ${ }^{10,11}$ systemic-to-pulmonary shunts, ${ }^{2,11-17}$ exercise physiology, ${ }^{18,19}$ cardiac biomechanics, ${ }^{20}$ an alternative initial palliation, ${ }^{21,22}$ and virtual surgery. ${ }^{23}$ Multiscale models require expertise in CFD to execute correctly, and they are computationally resource intensive, particularly in the clinical environment. This makes them cumbersome for use as an interactive bedside management tool or to use as an education tool to better explain SVP.

Therefore, we developed a simplified interactive simulator based on the LPM of these unique circulations, and we test their accuracy in estimating postoperative or postinterventional hemodynamics for all 3 stages of SVP. In essence, we trade off the detailed local hemodynamic information from the CFD simulations for a rapid, resource-available tool providing an accurate estimate of the circulation conditions in the various territories. Such a patient-specific clinical decision support tool can be disseminated as a Web-based tool or an application (app) on a hand-held device either to complement clinical management, to use as a visual aid in parent/patient counseling, and to assist in clinician/bioengineer education.

In LPM, the circulation is divided into several interconnecting compartments, each compartment comprising resistance, compliance, and inertial elements modeling the characteristics of the vasculature. For each compartment, there is an equation relating the volume of blood stored therein to the local transmural pressure. The rate of change of blood volume is equal to the net flow of blood in and out of the compartment. The compartments are linked by blood vessels, each characterized by its resistance to flow (mm $\mathrm{Hg} /[\mathrm{L} / \mathrm{min}])$. A compartment is a "lump" characterized by its pressure-volume relationship, which relates to the resistance and compliance within its vessels and to the inertia of the blood mass. The heart is a more sophisticated compartment, with the pressure-volume relationship changing significantly over the cardiac cycle. Overall, a well-designed LPM is a system of linked equations for pressures and flows that, when integrated, accurately simulates human circulation, including pulsatility. Such a model is 
computationally fast and requires only the modest resources available in hand-held devices. The model provides the kind of familiar measurements useful to understand health issues, where detailed velocity vector fields from CFD are not needed. Here we validate the utility of using LPM to predict pre- and postsurgical hemodynamics of the circulation.

\section{METHODS}

This simulation tool is based on the LPM approach to modeling the human circulation. ${ }^{24}$ The tool interface allows the user to supply available clinical data or to accept default generic values. We applied scaling rules to make the translation from user input values, which are typical clinical measurements, to the many internal parameters of the model. The simulation includes a fixed volume of blood in a closed-loop circulation, with a varying-compliance model of atrial and ventricular function tuned to match the specific case, and also a model of oxygen transport. The simulation presents clinical-style results to the user. In a final step, the user can explore the effects of changing inputs to the model or apply an exercise model. $^{18}$

\section{Simulation Workflow}

Simulation setup is divided into 3 sequential steps that define the patient and provide reasonable default values for the inputs (Video 1). A fourth step allows for modified simulations (Figure 1).

In Step 1, the user selects the circulation type. Options include modified Blalock-Taussig shunt (mBTS), Hybrid, or right ventricle to pulmonary artery (RV-PA) shunt for Stage 1 procedures; a superior cavopulmonary connection for Stage 2 procedures; and TCPC Fontan for Stage 3 procedures, in child or adult size.

In Step 2, the user sets the patient's body surface area (BSA). BSA is either specified directly or calculated from patient weight and height per Haycock and colleagues ${ }^{25}$ The BSA is used to assign default heart rate (HR), mean arterial and atrial pressures, and hemoglobin and to set the default ratio of upper to lower body flow.

In Step 3, the user sets the resting state of the patient. The mean arterial pressure (MAP) and single atrial pressure (SAP) entered here provide target values to tune the heart model. The patient-specific indexed systemic and pulmonary vascular resistance (SVRI, PVRI), resting oxygen consumption (vO2), HR, hemoglobin concentration, shunt diameter (for Stage 1 patients), or fenestration diameter (for Stage 3 patients) also can be entered. There are settings for aortic coarctation, venovenous collaterals, or aortopulmonary collaterals. Pulmonary artery stenosis diameters can be set, also serving as banded artery diameters in Hybrid Stage 1 circulation. An adjustment factor is provided for aortic arch compliance so as, for example, to simulate stiffening caused by scarring. Reasonable default values of all inputs are provided, and the user can accept them or replace them with measured values, or estimates.

In the background, the heart model is tuned to match the target MAP and SAP with respiration applied. Next, the simulation continues for the duration of 2 respiration cycles, and these results are presented in a table (eg, Figure 2, Baseline). Clinically relevant outcomes are presented, including systemic and pulmonary pressures, intracardiac pressures, cardiac index, pulmonary to systemic flow ratio (Qp:Qs), oxygen saturations, and systemic oxygen delivery. One of 6 heart diagrams that is appropriate for the given circulation is displayed, and pressure and flow plots are linked to the heart.

In Step 4, inputs used in Step 3 can be modified (aside from MAP or SAP, which become output parameters after the heart model has been set) to explore clinical changes or medical interventions. One may also change the circulation type or presence of a fenestration to predict postoperative hemodynamics. Additional input parameters, including respiratory

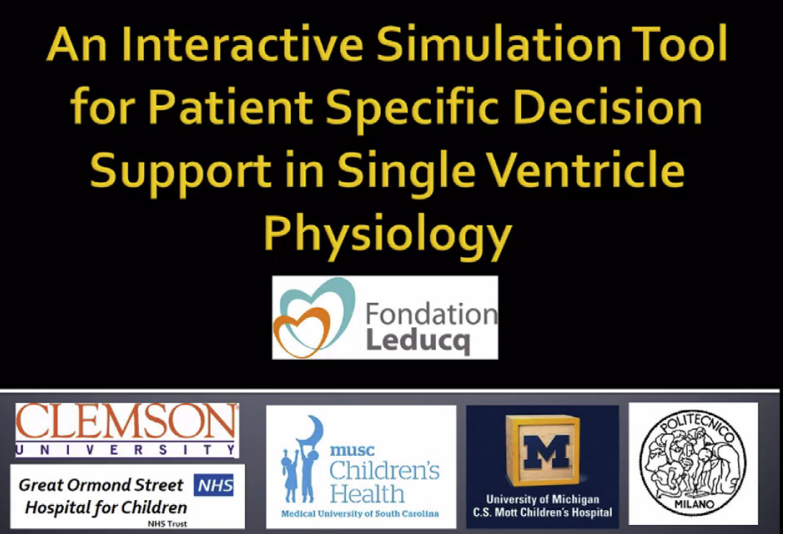

VIDEO 1. Example of using the simulation application: A video walkthrough. Video available at: http://www.jtcvsonline.org/article/S00225223(17)31963-3/fulltext.

rate, respiratory amplitude, and contractility, can be modified. Contractility is a coefficient applied to increase the systolic stiffness of the ventricle and the atrium. The contractility of the heart can be modified by inotropic medications, typically with a commensurate change of HR. The respiration input is the amplitude of intrathoracic pressure, which is negative in natural respiration. It can be changed to investigate respiratory effects on inferior vena cava and superior vena cava flows and on preload to the heart. The exercise protocol of Kung and colleagues ${ }^{18}$ can be invoked by setting the metabolic equivalent (MET). MET level 1 is the rest state. At MET levels 2 and greater, the HR and contractility, SVRI, PVRI, respiration rate, and amplitude are adjusted for exercise.

Figures 2 and 3 show an example of changing from mBTS (baseline) to Stage 2 superior cavopulmonary connection ("modified") circulation in Step 4. The MAP and SAP vary in response to the new inputs. As expected, the cardiac index decreases along with the Qp:Qs, and the systemic oxygen saturation and oxygen delivery increase. Clicking on an area of the heart diagram reveals pressure and flow plots (Figure 3), which are updated with the new simulation results.

\section{Circulation Model}

The circulation models originate from Corsini and colleagues, ${ }^{24}$ with circuit paths appropriately changed for each surgical stage (Figure 4). We added 3 optional resistive flow paths representing collaterals, 3 resistances representing aortic coarctation, and left and right pulmonary artery stenoses (or banding). Each Stage 1 circulation has a shunt model with quadratic resistance, each connected in a different way.

In the Step 3 simulation, all the resistance, compliance, and inertance elements are calculated using allometric equations on BSA. ${ }^{26}$ This gives the generic regional resistances: upper and lower body SVR, and right and left lung PVR. These are then compared with the given inputs for patient-specific SVR and ratio of upper body flow to lower body flow, and PVR. The resistances in each region are scaled directly by the necessary proportions. The compliances in each region are scaled according to the following equation,

$$
\frac{C}{C_{i}}=\left(\frac{R}{R_{i}}\right)^{-4 / 3}
$$

where $C / C_{\mathrm{i}}$ and $R / R_{\mathrm{i}}$ are the compliance and resistance scaling ratios. ${ }^{26}$

\section{Tuning the Frank-Starling Heart Model}

The heart model is matched to the resting state of the body. In modified simulations, HR and contractility can be adjusted to simulate response to 
Steps 1 and 2

Set Circulation Type and BSA.

Step 3

Enter patient-specific parameters as available: HR, MAP, SAP, PRVI, SVRI, shunt, Hb, fenestration, coarctation, collaterals, etc.

Step 4

Change virtual surgery and/or parameters:

HR, PVRI, SVRI, shunt, $\mathrm{Hb}$, fenestration, coarctation, collaterals, contractility, respiration, exercise, etc.
Tabulate typical patient parameters

for given Type and BSA.

Generate complete circulation model, and tune heart model to match inputs. Run simulation for baseline case.

Run new simulation for comparison.

FIGURE 1. In Step 3, the user makes any patient-specific changes to the given default values of rest-state parameters, and clicks "Set Baseline" to run the simulation. $B S A$, Body surface area; $H R$, heart rate; $M A P$, mean arterial pressure; $S A P$, single-atrium pressure; $P V R I$, indexed pulmonary vascular resistance; SVRI, indexed systemic vascular resistance.

stressors. But first, in the baseline case, a generic heart model appropriate for the selected single-ventricle circulation is adjusted and tuned to match patient-specific parameters. This is accomplished by stretching the enddiastolic pressure-volume relationship and end-systolic pressure-volume relationship curves. The simulation fine-tunes the model in run-time, using the errors of simulated MAP and SAP as feedback control signals, by adjusting the total blood volume in the circulation and heart size, until MAP and SAP approach the user-specified target values.

\section{Pressure-Volume Model}

Increasing the size (volume) of the heart can be treated as producing the same range of pressures over an increased range of volume. Starting from a standard heart model, it is convenient to scale it up by scaling down the volume, which is an input to all the pressure equations. In defining the scaled volume $D V_{\mathrm{sv}}$, the actual single-ventricle volume $V_{\mathrm{sv}}$ is scaled around the unstressed volume $V_{\mathrm{Sv} 0}$, and the size factor $f_{\text {size }}$ is initially proportionate to the desired stroke volume:

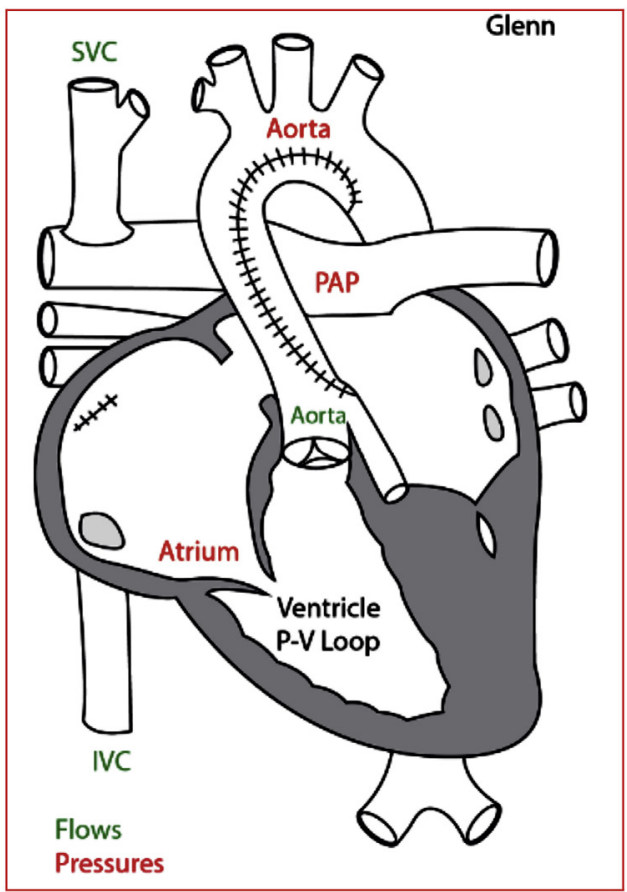

\begin{tabular}{|lll|}
\hline Parameter & Baseline & Modified \\
\hline COI & 5.51 & 3.67 \\
\hline Qp/Qs & 1.32 & 0.65 \\
\hline Aorta & $94 / 20 / 54 / 78 \%$ & $115 / 53 / 82 / 89 \%$ \\
\hline Ventricle & $94 / 12.9 / 78 \%$ & $115 / 10.2 / 89 \%$ \\
\hline Atrium & $9.6 / 3.4 / 6.9 / 78 \%$ & $6.9 / 1.6 / 4.7 / 89 \%$ \\
\hline PulmArt & $16.8 / 10.0 / 14.1 /$ & $11.3 / 8.3 / 10.1 / 7$ \\
\hline SVC & $78 \%$ & $2 \%$ \\
\hline IVC & $0.47 / 54 \%$ & $0.71 / 72 \%$ \\
O2DeliveryI & 411 & $0.39 / 73 \%$ \\
\hline
\end{tabular}

$$
\begin{aligned}
& \text { more results } \\
& \hline \text { download baseline } \\
& \hline \text { download modified } \\
& \hline
\end{aligned}
$$

FIGURE 2. The results of the baseline and modified simulations are displayed in a table, along with the appropriate heart diagram. Clicking on any of the labels in the heart sketch displays a plot of pressure or flow. In this example, the circulation has been changed from mBT (Baseline) to Stage 2 superior cavopulmonary connection (Modified). Note that there is a decrease in the COI and Qp:Qs, but an increase in the systemic oxygen saturation and indexed oxygen delivery. Aorta, atrium, and pulmonary artery parameters: systolic/diastolic/mean pressures/oxygen saturation. Ventricle parameter: systolic/enddiastolic pressures/oxygen saturation. SVC and IVC parameters: flow in L/min and oxygen saturation. COI, Cardiac output index; $Q p: Q s$, pulmonary to systemic flow ratio; $S V C$, superior vena cava; $P A P$, pulmonary arterial pressure; $I V C$, inferior vena cava. 

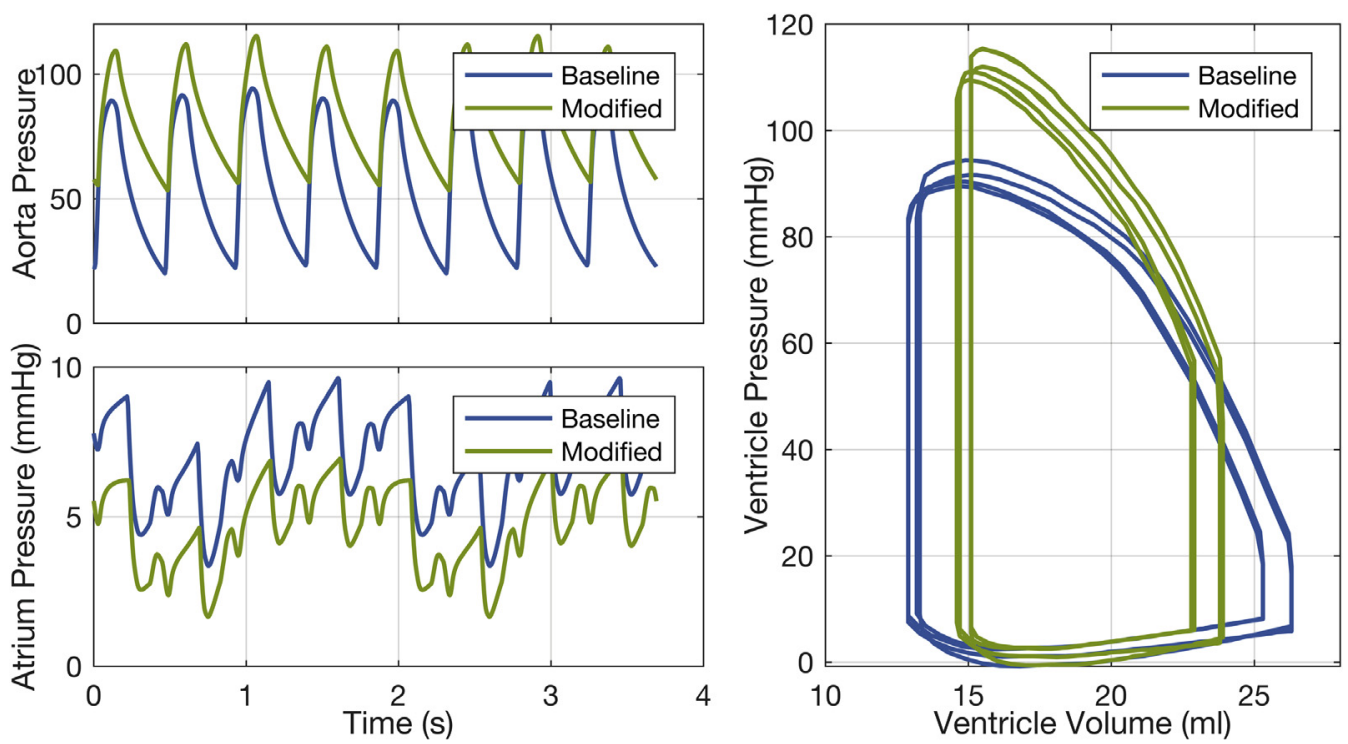

FIGURE 3. Example of plots resulting from the baseline and modified runs. The ventricular pressure-volume loops in the upper left plot show the expected changes with change from mBTS (Baseline) to Stage 2 (Modified) circulation. The upper right plot shows the aortic pressure, and the lower plot is the atrial pressure.

$$
D V_{s v}=\left(V_{s v}-V_{s v 0}\right) / f_{\text {size }}
$$

The end-diastolic pressure-volume relationship is as follows:

$$
P_{s v, p a s s i v e}=f_{D} \cdot \operatorname{csv}\left(e^{d s v \cdot D V_{s v}}-1+2 d s v \cdot D V_{s v}\right)
$$

where $c s v$ and $d s v$ are constants from the standard design and $f_{\mathrm{D}}$ is proportionate to target SAP. The end-systolic pressure-volume relationship is as follows,

$$
P_{s v, \text { active }}=f_{C} \cdot f_{S} \cdot E_{\max } \cdot D V_{s v}
$$

a straight line characterized by the maximum stiffness $E_{\max } \cdot f_{\mathrm{S}}$ is proportionate to target MAP, and $f_{\mathrm{C}}$ is the user-defined factor for increased contractility in Step 4.

The normalized elastance $E_{\mathrm{N}}(t)$ is an activation function used to interpolate between the passive and active curves. ${ }^{27}$ The instantaneous transmural pressure is as follows:

$$
S V P=E_{N}(t) P_{s v, a c t i v e}+P_{s v, p a s s i v e}
$$

Finally, the effective ventricular pressure is modified by the myocardial flow resistance, and by the intrathoracic pressure $P_{\text {th }}$.

$$
P_{s v, e f f}=S V P-R_{m y o} Q_{A o}+P_{t h}
$$

Thus, respiration directly affects ventricular pressure. The atrium pressure model is similar in nature. ${ }^{18}$ The model for flow through the atrioventricular valve includes inertia. The aortic valve is modeled as a square-law resistance with perfect closure and zero inertia.

\section{Oxygen Transport}

Oxygen transport and oxygen saturations are calculated via the mean arterial and venous flow rates from the simulation. Pulmonary venous saturation is maintained at $97 \%$. Hemoglobin level $(\mathrm{g} / \mathrm{dL})$ can be changed between Steps 3 and 4. Blood saturation in the heart is diluted according to the various different circulations. Oxygen delivery is indexed by BSA:

$$
\mathrm{O}_{2} \text { DeliveryI }=10 C_{\text {Aorta }} Q s / B S A,
$$

in $\mathrm{mL}_{\mathrm{O} 2} / \mathrm{min} / \mathrm{m}^{2}$. $C_{\text {Aorta }}$ is the concentration of oxygen in the aorta $(\mathrm{mL} / \mathrm{dL}$ ), and $Q s$ is systemic flow (in $\mathrm{L} / \mathrm{min}$ ), which is less than cardiac output in some circulations. Oxygen consumption is an input in both Steps 3 and 4, and the default value is vO2 $=160 \mathrm{mLO}_{2} /$ $\mathrm{min} / \mathrm{m}^{2}$ (the same units as delivery). Effects of shunts, fenestration, and collaterals are accounted for. For Stage 2 and 3 models, systemic venous-to-pulmonary venous collaterals are accounted for by connection between the superior vena cava (and inferior vena cava in the Stage 3 model) to the right pulmonary vein, diluting the saturation there from $97 \%$. Aorto-to-pulmonary arterial collaterals are considered as the connection between the aorta and left pulmonary artery. The magnitude of both types of collateralization can be varied by the user to examine their influences on various output parameters, such as systemic arterial saturation ( $\mathrm{SaO} 2)$, Qs, and oxygen delivery.

\section{Respiration Pressures}

Respiration effects are modeled by varying the external pressure on those LPM compliance elements located within the thoracic cavity or the abdominal cavity. The waveform of each cavity pressure is a sinusoid with a rest. In the thoracic cavity, respiration amplitude is negative, except it is positive during forced ventilation. In the abdominal cavity, the amplitude is positive. In Step 3, default respiration values are assigned. The respiration rate is fixed at one quarter the HR. ${ }^{18}$ In Step 4, respiration rate and amplitude can be changed.

\section{Validation}

Although many of the model parameters are directly matched to patient measurements, some others are based on correlations and thus are subject to validation testing on resulting outputs. These include the performance of the shunt in Stage 1, the ratio of upper body flow to lower body flow, fenestration, and the oxygen transport model.

Catheterizations at various stages were obtained from 47 patients with SVP who were enrolled in a larger multi-institutional study by the Modeling of Congenital Hearts Alliance (MOCHA) investigators. ${ }^{28}$ The study was approved by each site's institutional review board, and informed consent was obtained for all patients. This study included children and adults with various forms of SVP, including hypoplastic left heart syndrome, tricuspid atresia, pulmonary atresia with intact ventricular septum, and double-inlet left ventricle. Exclusion criteria included atrial isomerism, an interrupted inferior vena cava, anomalous pulmonary venous connections, or an intact atrial septum. 


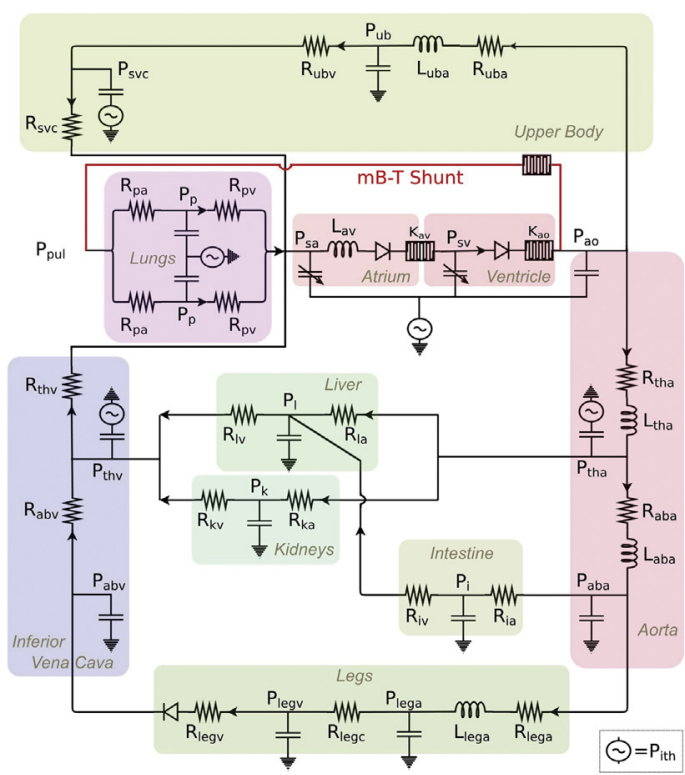

A

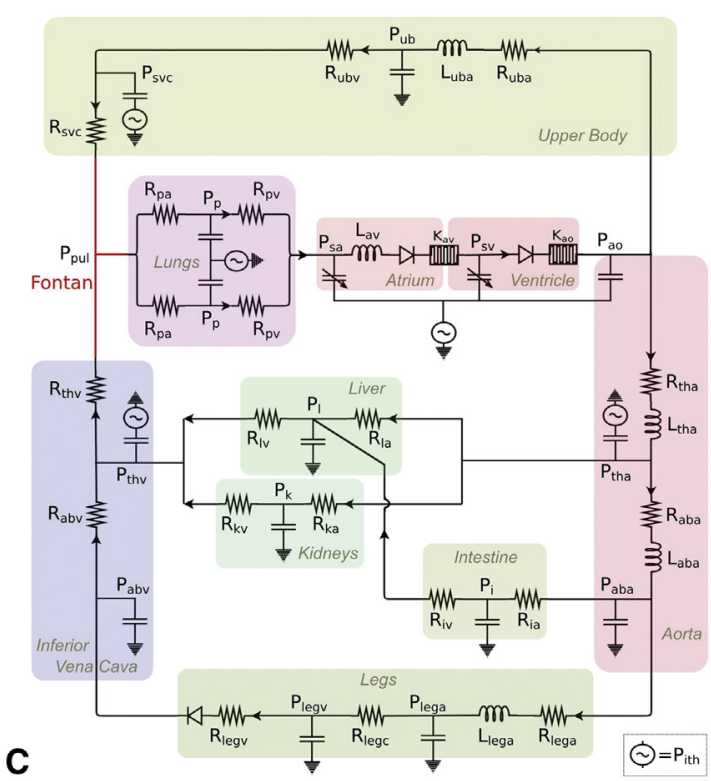

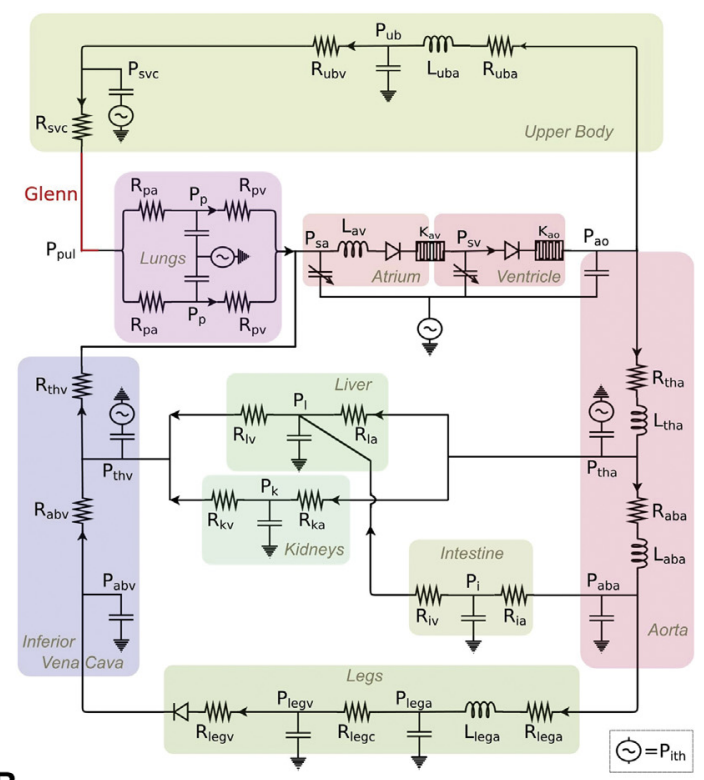

B

FIGURE 4. Schematics of the lumped parameter network models for each stage of single-ventricle physiology. A, Stage 1 circulation with modified Blalock-Taussig shunt. B, Stage 2 Glenn circulation model. C, Fontan circulation model.

As the result of institutional preferences, the vast majority of Stage 1 patients enrolled in the larger study had a mBTS rather than a RV-PA shunt or Hybrid. As a result, there was inadequate power to validate the RV-PA shunt or Hybrid models. Therefore, the Stage 1 patients in this study were limited to those with mBTS. Stage 2 patients included both HemiFontan and bidirectional Glenn connection patients, given that the LPM does not differentiate the geometric/anatomic differences between these 2 different surgical techniques for superior cavopulmonary connection. Similarly, both lateral tunnel and extracardiac conduit TCPC Fontan patients were included in Stage 3 validation.

Data were collected from 60 catheterization reports performed on the 47 patients, as some of them had catheterizations for different stages. For the validation study, 20 catheterization reports $(n=20)$ were adopted for each surgical stage. The characteristics of these patients are given in Table 1. In the Stage 1 group, most of the patients had a 4-mm shunt ( $3 \mathrm{~mm}: 1 ; 3.5 \mathrm{~mm}$ :
4; $4 \mathrm{~mm}: 14 ; 5 \mathrm{~mm}: 1$ ). All of the Stage 2 patients had a bidirectional Glenn connection. Only 1 Stage 3 patient had a lateral tunnel Fontan, whereas the rest had an extracardiac TCPC. At Stage 3, 12 of 20 patients had a patent fenestration.

The catheterization reports were reviewed, and the following information was recorded: SAP, MAP, BSA, HR, hemoglobin, assumed vO2, PVRI, and SVRI. Each patient's information was entered into the online model, and the baseline output results were recorded. The following information was compared between the model predictions and clinical measurements (from the catheterization report): Qp:Qs, $\mathrm{SaO} 2$, mean pulmonary artery pressure (mPAp), and systemic venous saturation ( $\mathrm{SvO} 2)$. The oxygen saturation from the superior vena cava was used as the $\mathrm{SvO} 2$ for both the simulation model and the catheterization. The catheterization results were compared with the model results via a paired Student $t$ test. A linear regression was performed for each output variable equating the 
TABLE 1. Baseline patient characteristics for each stage (average \pm standard deviation)

\begin{tabular}{|c|c|c|c|c|c|c|}
\hline Stage & Height, cm & Weight, kg & BSA, $\mathbf{m}^{2}$ & PVRI, WU $\cdot \mathbf{m}^{2}$ & SVRI, WU $\cdot \mathbf{m}^{2}$ & Mean SAP, mm Hg \\
\hline 1 & $65 \pm 6$ & $6.2 \pm 1.5$ & $0.33 \pm 0.05$ & $2.16 \pm 0.89$ & $19.0 \pm 8.4$ & $8 \pm 2$ \\
\hline 2 & $95 \pm 14$ & $15.5 \pm 6.5$ & $0.63 \pm 0.17$ & $1.99 \pm 1.02$ & $10.5 \pm 3.7$ & $8 \pm 2$ \\
\hline 3 & $132 \pm 33$ & $36 \pm 24.8$ & $1.1 \pm 0.53$ & $1.75 \pm 1.04$ & $14.6 \pm 5.0$ & $9 \pm 3$ \\
\hline
\end{tabular}

$\mathrm{N}=20$ for each stage. $B S A$, Body surface area; $P V R I$, indexed pulmonary vascular resistance; $W U$, Wood Units; $S V R I$, indexed systemic vascular resistance; SAP, single-atrium pressure.

catheterization result to the model result, and a Bland-Altman analysis also was performed. $^{29}$

\section{RESULTS}

Catheterization results are compared with simulation results in Table 2. There were no significant differences between the catheterization results and model output for Stage 1 patients in regard to average Qp:Qs, mPAp, or $\mathrm{SaO} 2(P>.38)$. There was a statistically significant difference in average $\mathrm{SaO} 2-\mathrm{SvO} 2$, but this difference was not clinically significant $(26 \%$ vs $27 \%, P<.001)$. Among Stage 2 patients, there were no significant differences between the catheterization results and model output with regard to average Qp:Qs, mPAp, or $\mathrm{SaO} 2(P>.09)$, but a statistically significant difference existed in average $\mathrm{SaO} 2-\mathrm{SvO} 2(17 \%$ vs $19 \%, P<.001)$. Among Stage 3 patients, there were no significant differences between the catheterization results and model output with regard to average Qp:Qs, mPAp, $\mathrm{SaO} 2$, or $\mathrm{SaO} 2-\mathrm{SvO} 2(P>.2)$.

When we evaluated the group as a whole, there were no significant differences in average Qp:Qs, $\mathrm{SaO} 2$, or mPAp. There remained a statistically significant difference in average $\mathrm{SaO} 2-\mathrm{SvO} 2$, but this was not clinically significant $(20 \%$ vs $21 \%, P=.014)$. The linear regression analyses (Figure 5) revealed good predictive value of the model for each variable $\left(\mathrm{Qp}: \mathrm{Qs}, \mathrm{R}^{2}=0.79 ; \mathrm{SaO} 2, \mathrm{R}^{2}=0.64\right.$;
mPAp, $\left.\mathrm{R}^{2}=0.69 ; \mathrm{SaO} 2-\mathrm{SvO} 2, \mathrm{R}^{2}=0.93\right)$. The BlandAltman analysis (Figure 6) revealed that the average difference between the measurements made by the model and catheterization were close to zero (mean difference $\mathrm{Qp}: \mathrm{Qs}=0.0045, \mathrm{SaO} 2=-0.2 \%, \mathrm{mPAp}=0.057, \mathrm{SaO} 2-$ $\mathrm{SvO} 2=-0.65)$, which is consistent with the $t$-test results. This shows that there was no evidence of a systematic difference (fixed bias) between the results provided by catheterization versus the model. Thus, the average results from the model matched very closely with the catheterization measurements. The Bland-Altman plots also provide a graphical representation of the variability in individual measurements, with $95 \%$ confidence intervals. The confidence intervals on these plots reveal that the spread of data does reach a clinically significant difference for some patients.

\section{DISCUSSION}

We have adopted lumped parameter methodology to develop an intuitive simulation tool that is user-friendly and capable of producing patient-specific output information rapidly with good clinical accuracy. Unlike previously published mathematical or computational models, this selfcontained tool does not require specialized computational software and can be executed through a Web browser or on an iOS device. In addition, it is designed for use by

TABLE 2. Comparison of the catheterization results with the simulation model predictions for each surgical stage

\begin{tabular}{|c|c|c|c|c|}
\hline Stage & Catheterization, mean \pm SD & Simulation tool, mean \pm SD & Mean difference & $P$ value \\
\hline \multicolumn{5}{|l|}{$1(\mathrm{n}=20)$} \\
\hline Qp:Qs & $1.4: 1 \pm 0.7$ & $1.4: 1 \pm 0.6$ & -0.03 & .69 \\
\hline $\mathrm{SaO} 2, \%$ & $75 \pm 7$ & $76 \pm 6$ & -1.25 & .36 \\
\hline mPAp, mm Hg & $15 \pm 3$ & $16 \pm 3$ & -0.4 & .38 \\
\hline $\mathrm{SaO} 2-\mathrm{SvO} 2, \%$ & $26 \pm 9$ & $27 \pm 9$ & -0.7 & $<.001$ \\
\hline \multicolumn{5}{|l|}{$2(\mathrm{n}=20)$} \\
\hline Qp:Qs & $0.6: 1 \pm 0.1$ & $0.5: 1 \pm 0.04$ & 0.04 & .06 \\
\hline $\mathrm{SaO} 2, \%$ & $82 \pm 5$ & $81 \pm 5$ & 1.5 & .34 \\
\hline mPAp, mm Hg & $13 \pm 3$ & $13 \pm 3$ & 0.5 & .09 \\
\hline $\mathrm{SaO} 2-\mathrm{SvO} 2, \%$ & $17 \pm 5$ & $19 \pm 5$ & -1.5 & $<.001$ \\
\hline \multicolumn{5}{|l|}{$3(\mathrm{n}=20)$} \\
\hline Qp:Qs & $0.9: 1 \pm 0.2$ & $0.9: 1 \pm 0.1$ & $<0.01$ & .98 \\
\hline $\mathrm{SaO} 2, \%$ & $93 \pm 5$ & $94 \pm 3$ & -0.85 & .21 \\
\hline mPAp, mm Hg & $14 \pm 2$ & $14 \pm 3$ & 0.1 & .63 \\
\hline $\mathrm{SaO} 2-\mathrm{SvO} 2, \%$ & $26 \pm 7$ & $26 \pm 8$ & 0.25 & .70 \\
\hline
\end{tabular}

The "mean difference" is the average difference in individual values (catheterization result - simulation tool). $S D$, Standard deviation; $Q p$ : $Q s$, pulmonary to systemic flow ratio; $\mathrm{SaO}$, arterial blood saturation; $m P A$, mean pulmonary artery pressure; $\mathrm{SvO}$, venous blood saturation, measured at the superior vena cava. 

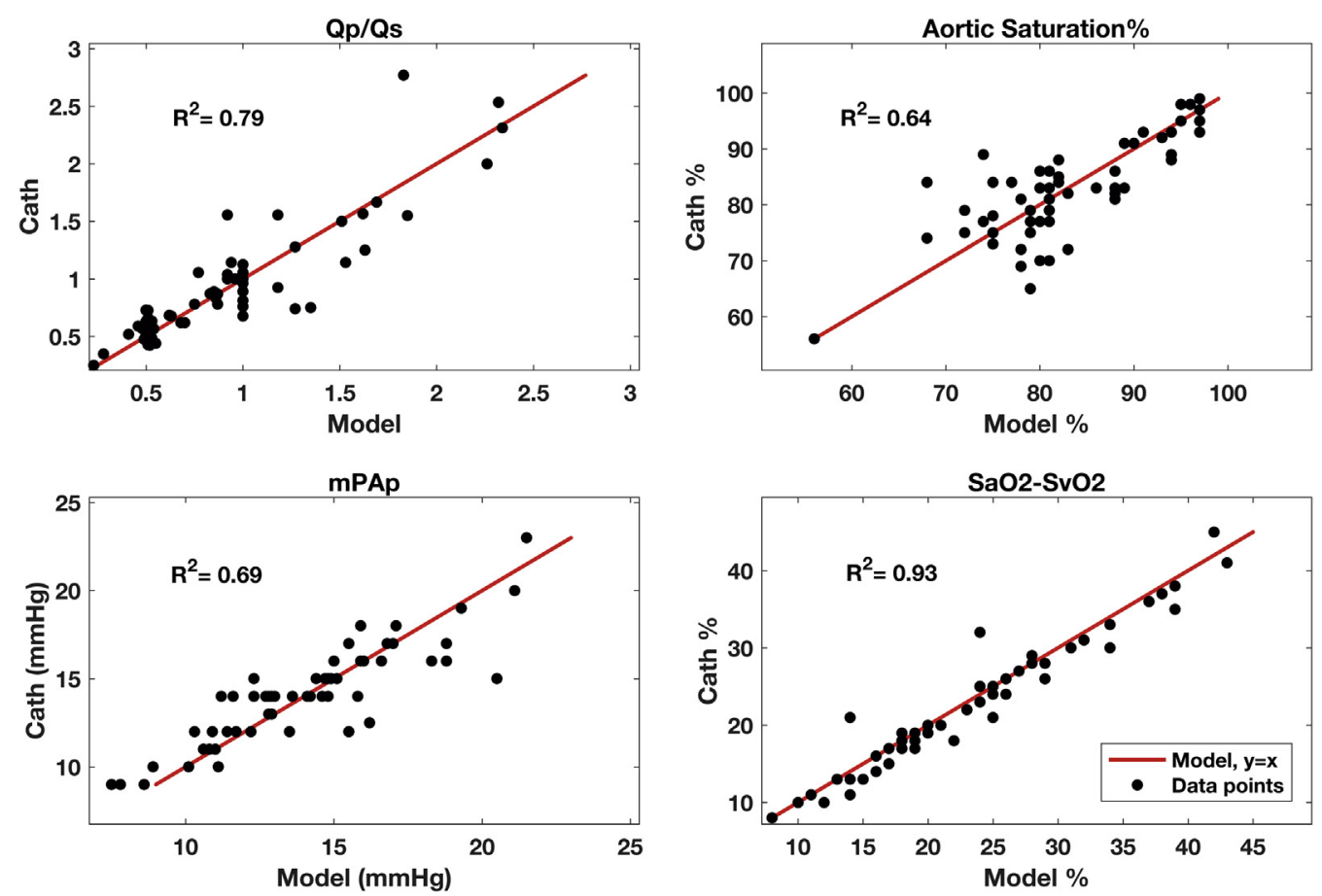

FIGURE 5. Linear regression analyses for catheterization and model output variables. $Q p: Q s$, Pulmonary to systemic flow ratio; $m P A p$, mean pulmonary artery pressure; $\mathrm{SaO} 2-\mathrm{SvO}$, difference between arterial saturation and venous saturation.

clinicians without the need for significant knowledge or training in engineering and by engineers without need for in-depth understanding of the pathophysiology and anatomic derangements in the various forms of SVP. The encouraging initial validation results described here demonstrate that modeling of SVP in a clinical decision support
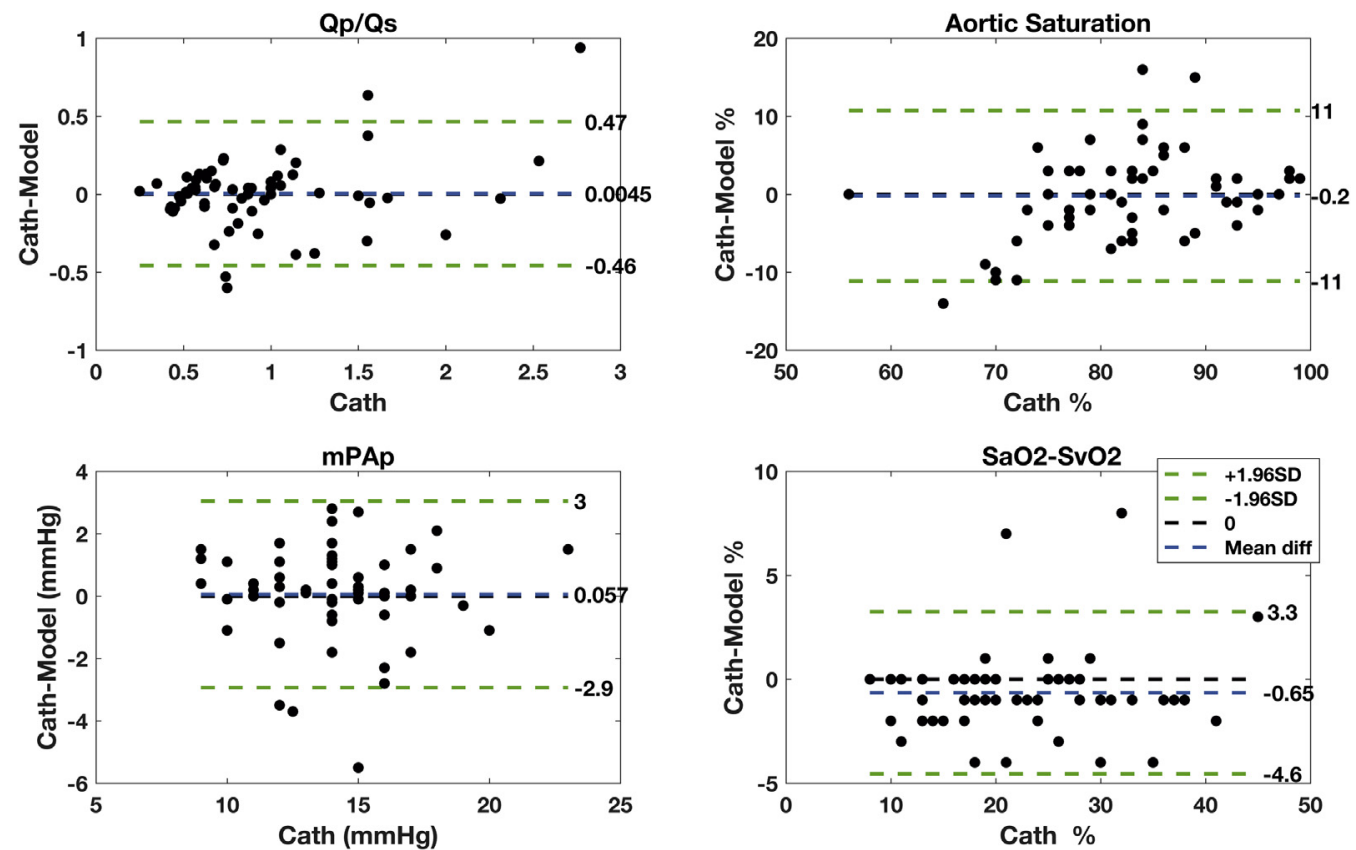

FIGURE 6. Bland-Altman analyses for catheterization and model output variables. The vertical axis is the difference between the catheterization result and the model prediction, and the horizontal axis is the catheterization result. $Q p: Q s$, Pulmonary to systemic flow ratio; $m P A p$, mean pulmonary artery pressure; $\mathrm{SaO} 2-\mathrm{SvO} 2$, difference between arterial saturation and venous saturation. 
system can benefit a larger community beyond the few engineering/clinical collaborators, such as in bedside education and clinician-patient dialogue.

Although the model provides good clinical accuracy for most patients enrolled in this validation, the Bland-Altman analyses revealed clinically significant variability in the results. The potential cause of this variation is multifactorial. First, one must consider the inherent variability in measurements made in the catheterization laboratory. Although catheterization is considered the "gold standard" for hemodynamic measurements in these patients, it is known that the standard deviation of repeated mixed venous saturation measurements in a single patient is $\sim 3 \% .{ }^{30}$ As a result, the $95 \%$ confidence level for mixed venous saturation measurements in the catheterization laboratory is $\pm 6 \%$. This could explain more than half of the difference seen in the saturation measurements in our patients. Given that saturation measurements are the sole determinant for Qp:Qs calculations made in the catheterization laboratory (after other variables cancel each other out), a portion of the variability in Qp:Qs measurements in our results is potentially explained by this as well. Furthermore, given that many of these patients were under general anesthesia (often with positive pressure ventilation) for the catheterization, it is reasonable to conclude that their hemodynamic state is at least slightly deviated from baseline, with potential dynamic changes in pressure and saturation measurements. The discrepancy also could have resulted from parameters not included in the catheterization report, such as contractility, arterial compliance, respiratory rate, or respiratory amplitude. Venovenous and aortopulmonary collaterals can have a significant effect on hemodynamics, but in the catheterization reports, their significance is generally measured only subjectively, if at all. Subtle abnormalities such as mild/diffuse shunt stenosis or mild pulmonary artery hypoplasia, which might not be noted or quantified on the catheterization report, can create discrepancies as well. Also, it is possible that some measurements taken during the catheterization were not taken simultaneously or were taken under abnormal clinical conditions. When the baseline simulation on the model does not match the patient's clinical results, the user can consider possible reasons for discrepancies and then make appropriate adjustments in the inputs to match the patient's measured physiology.

Unlike CFD modeling, the LPM does not consider patient-specific 3-dimensional anatomy of the surgically altered domain, so it cannot examine more complex scenarios, such as the local differences between a bidirectional Glenn versus Hemi-Fontan. However, most common clinical scenarios and interventions can be simulated using this tool. For instance, clinicians can demonstrate the physiologic response to medical modulation of SVR or PVR, fenestration closure, or blood transfusion. The hemodynamic benefit of an intervention on pulmonary artery stenosis, aortic coarctation, venovenous collaterals, or aortopulmonary collaterals also can be predicted. Used in combination with conventional investigations, such as magnetic resonance imaging and catheterization data, the simulation tool can help to assess the risk stratification for progression to the next surgical stage or to quantitatively examine the effects of various patient management decisions and as such provide a clinical tool to examine interventional strategies with anticipated outcomes.

Another potentially important use of this simulation tool is in parent/patient education and counseling. For most parents of children with single-ventricle heart defects, the concept and physiologic consequence of SVP palliative management can be challenging to grasp. Therefore, the simulation tool can assist cardiologists and surgeons to graphically and quantitatively describe the rationale and expected outcomes of the 3-stage surgical palliation or the benefits and indications of a specific intervention being recommended. Moreover, in cases in which the next surgical stage presents important risks, such as in cases in which pre-Fontan investigations showed a borderline candidacy for Fontan completion, the simulator can be valuable for assisting clinicians in explaining to the parents why the Fontan operation is potentially risky and what interventions can be used to mitigate against postoperative complications.

The SVP simulator can be an important adjunct in education and training of cardiology and surgical trainees. Combined with bedside teaching on rounds or conferences, and available on an iOS device, the simulator can be useful to aid understanding of the dramatic changes in cardiovascular and pulmonary physiology introduced by all 3 stages of SVP palliation. Similarly, the simulator can visually demonstrate relationships between medical and/or surgical manipulation and response by clinically familiar variables. This tool could increase the focus of training on the physics of circulation, potentially changing the way that clinical trainees think about SVP.

In addition to the aforementioned limitations, it should be noted that we lacked an adequate number of patients to perform a validation of the models for the Stage 1 RV-PA shunt or hybrid procedure. However, because our model is based on validated computational models of these circulations that have been previously published, ${ }^{8,12-15}$ we speculate that our models of these 2 circulations should behave with similar accuracy to the mBTS results. As more clinical data become available, we can test this hypothesis adequately.

Lastly, the role of the SVP simulator is to provide an additional tool to support clinicians in managing their patients by estimating hemodynamic and physiologic outcomes before an operation or intervention. As no mathematical or computational modeling or simulation study can account for all the biological processes and system variables that can influence outcome, the SVP 
simulator is intended as an advisory or training tool and not as an instrument that will prognosticate or predict patient outcomes.

\section{CONCLUSIONS}

With the use of validated lumped parameter methods, complete cardiopulmonary circulatory models of each surgical stage of single-ventricle palliation were translated into a simulator that can be used to support clinical decisions. This simulator produces patient-specific outputs with good clinical accuracy. It can be used by clinicians anywhere on computers or mobile devices and requires no previous knowledge of engineering modeling. In addition to estimating postoperative hemodynamics, the simulator can support clinical decision-making by quantitatively assessing questions such as shunt sizes and fenestration closure and effects of modulating PVRs or SVRs or closing collaterals.

\section{Conflict of Interest Statement}

Authors have nothing to disclose with regard to commercial support.

\section{References}

1. DiBardino DJ. Long-term progression and survival following Norwood Single Ventricle Reconstruction. Curr Opin Cardiol. 2015;30:95-9.

2. Migliavacca F, Pennati G, Dubini G, Fumero R, Pietrabissa R, Urcelay G, et al. Modeling of the Norwood circulation: effects of shunt size, vascular resistances, and heart rate. Am J Physiol Heart Circ Physiol. 2001;280:H2076-86.

3. Slesnick TC, Yoganathan AP. Computational modeling of Fontan physiology: at the crossroads of pediatric cardiology and biomedical engineering. Int J Cardiovasc Imaging. 2014:30:1073-84.

4. Watrous RL, Chin AJ. Model-based comparison of the normal and Fontan circulatory systems: part i: development of a general purpose, interactive cardiovascular model. World J Pediatr Congenit Heart Surg. 2014;5:372-84.

5. Ceballos A, Argueta-Morales IR, Divo E, Osorio R, Caldarone CA, Kassab AJ, et al. Computational analysis of hybrid Norwood circulation with distal aortic arch obstruction and reverse Blalock-Taussig shunt. Ann Thorac Surg. 2012; 94:1540-50.

6. de Vecchi A, Nordsletten DA, Remme EW, Bellsham-Revell H, Greil G, Simpson JM, et al. Inflow typology and ventricular geometry determine efficiency of filling in the hypoplastic left heart. Ann Thorac Surg. 2012;94:1562-9.

7. Qian Y, Liu JL, Itatani K, Miyaji K, Umezu M. Computational hemodynamic analysis in congenital heart disease: simulation of the Norwood procedure. Ann Biomed Eng. 2010;38:2302-13.

8. Baker CE, Corsini C, Cosentino D, Dubini G, Pennati G, Migliavacca F, et al. Effects of pulmonary artery banding and retrograde aortic arch obstruction on the hybrid palliation of hypoplastic left heart syndrome. J Thorac Cardiovasc Surg. 2013;146:1341-8.

9. Schiavazzi DE, Kung EO, Marsden AL, Baker C, Pennati G, Hsia TY, et al. Hemodynamic effects of left pulmonary artery stenosis after superior cavopulmonary connection: a patient-specific multiscale modeling study. J Thorac Cardiovasc Surg. 2015;149:689-96. e1-e3.

10. Biglino G, Corsini C, Schievano S, Dubini G, Giardini A, Hsia TY, et al. Computational models of aortic coarctation in hypoplastic left heart syndrome: considerations on validation of a detailed 3D model. Int J Artif Organs. 2014;37:371-81.

11. Corsini C, Biglino G, Schievano S, Hsia TY, Migliavacca F, Pennati G, et al. The effect of modified Blalock-Taussig shunt size and coarctation severity on coronary perfusion after the Norwood operation. Ann Thorac Surg. 2014;98: 648-54.

12. Biglino G, Giardini A, Baker C, Figliola RS, Hsia TY, Taylor AM, et al. Implementing the Sano modification in an experimental model of first-stage palliation of hypoplastic left heart syndrome. ASAIO J. 2013;59:86-9.

13. Biglino G, Giardini A, Ntsinjana HN, Schievano S, Hsia TY, Taylor AM. Ventriculoarterial coupling in palliated hypoplastic left heart syndrome: noninvasive assessment of the effects of surgical arch reconstruction and shunt type. $J$ Thorac Cardiovasc Surg. 2014;148:1526-33.

14. Bove EL, Migliavacca F, de Leval MR, Balossino R, Pennati G, Lloyd TR, et al Use of mathematic modeling to compare and predict hemodynamic effects of the modified Blalock-Taussig and right ventricle-pulmonary artery shunts for hypoplastic left heart syndrome. J Thorac Cardiovasc Surg. 2008;136:312-20.e2.

15. Hsia TY, Migliavacca F, Pennati G, Balossino R, Dubini G, de Leval MR, et al Management of a stenotic right ventricle-pulmonary artery shunt early after the Norwood procedure. Ann Thorac Surg. 2009;88:830-7; discussion 83 7-8.

16. Migliavacca F, Dubini G, Pennati G, Pietrabissa R, Fumero R, Hsia TY, et al Computational model of the fluid dynamics in systemic-to-pulmonary shunts. J Biomech. 2000;33:549-57.

17. Moghadam ME, Migliavacca F, Vignon-Clementel IE, Hsia TY, Marsden AL. Optimization of shunt placement for the Norwood surgery using multi-domain modeling. J Biomech Eng. 2012;134:051002.

18. Kung E, Pennati G, Migliavacca F, Hsia TY, Figliola R, Marsden A, et al. A simulation protocol for exercise physiology in Fontan patients using a closed loop lumped-parameter model. J Biomech Eng. 2014;136.

19. Kung E, Perry JC, Davis C, Migliavacca F, Pennati G, Giardini A, et al. Computational modeling of pathophysiologic responses to exercise in Fontan patients. Ann Biomed Eng. 2015;43:1335-47.

20. Meoli A, Cutri E, Krishnamurthy A, Dubini G, Migliavacca F, Hsia TY, et al. A multiscale model for the study of cardiac biomechanics in single-ventricle surgeries: a clinical case. Interface Focus. 2015;5:20140079.

21. Esmaily-Moghadam M, Hsia TY, Marsden AL. The assisted bidirectional Glenn: a novel surgical approach for first-stage single-ventricle heart palliation. J Thorac Cardiovasc Surg. 2015;149:699-705.

22. Zhou J, Esmaily-Moghadam M, Hsia TY, Marsden AL, Figliola RS. In vitro assessment of the assisted bidirectional Glenn for stage 1 single-ventricle repair. Cardiovasc Eng and Tech. 2015;6:256-67.

23. Corsini C, Baker C, Baretta A, Biglino G, Hlavacek AM, Hsia TY, et al Integration of clinical data collected at different times for virtual surgery in single ventricle patients: a case study. Ann Biomed Eng. 2015;43: 1310-20.

24. Corsini C, Baker C, Kung E, Schievano S, Arbia G, Baretta A, et al. An integrated approach to patient-specific predictive modeling for single ventricle heart palliation. Comput Methods Biomech Biomed Engin. 2014;17:1572-89.

25. Haycock GB, Schwartz GJ, Wisotsky DH. Geometric method for measuring body surface area: a height-weight formula validated in infants, children and adults. J Pediatr. 1978;93:62-6.

26. Baretta A, Corsini C, Yang W, Vignon-Clementel IE, Marsden AL, Feinstein JA, et al. Virtual surgeries in patients with congenital heart disease: a multiscale modelling test case. Philos Trans A. Math Phys Eng Sci. 2011;369:4316-30.

27. Senzaki H, Chen CH, Kass DA. Single-beat estimation of end-systolic pressurevolume relation in humans. A new method with the potential for noninvasive application. Circulation. 1996;94:2497-506.

28. Hsia TY, Figliola RS. Multiscale modeling of single ventricle hearts for clinical decision support: a Leducq Transatlantic Network of Excellence. Eur J Cardiothoracic Surg. 2016;49:365-8.

29. Bland JM, Altman DG. Statistical methods for assessing agreement between two methods of clinical measurement. Lancet. 1986;1:307-10.

30. Freed MD, Miettinen OS, Nadas AS. Oximetric detection of intracardiac left-toright shunts. Br Heart J. 1979;42:690-4.

Key Words: single ventricle physiology, computer modeling and simulation, clinical decision support system, fontan circulation, Glenn or superior cavopulmonary circulation 